\title{
Well-being inequality and reference groups: an agenda for new research
}

\author{
Bernard Van Praag
}

Received: 13 January 2010 / Accepted: 13 January 2010 / Published online: 10 February 2010

(C) The Author(s) 2010. This article is published with open access at Springerlink.com

\begin{abstract}
In this paper it is argued that subjective well-being (SWB) of the individual depends on two types of variables. The first type consists of characteristics of the individual himself, such as age, health, income, etc. The second type of variables consists of the characteristics of the individuals belonging to his reference group. The vast literature about happiness, quality of life, and well-being informs us extensively about the effects of objective variables. How the second type affects well-being is much less investigated. It is argued that the concept of well-being inequality cannot be properly defined without taking the referencing process into account. The reference effect depends on how frequently individuals compare with others and on the degree of social transparency in society. We attempt to give a structural embedding of the idea of reference groups in SWB-models. In this paper we employ the reference-extended model for incorporating in happiness studies the concept of inequality in happiness or SWB. Finally, we plead for an extension of the present happiness paradigm by setting up a new additional agenda for empirical research in order to get quantified knowledge about the referencing process. As a first step we suggest a new question module to be included in new survey questionnaires.
\end{abstract}

Keywords Subjective well being $\cdot$ Happiness $\cdot$ Inequality $\cdot$ Reference group

\section{Introduction}

In the body of traditional economics the role of the utility concept has been ambiguous. On the one hand it is central in micro-economic model building to explain human choice behavior. On the other hand it is shunned, since it appears very hard to give it an empirical content. With respect to the explanation of choice behavior

B. Van Praag $(\varangle)$

Tinbergen Institute, University of Amsterdam, CESifo, IZA, DIW, Amsterdam, The Netherlands e-mail: b.m.s.vanpraag@uva.nl 
this could be overcome by introducing the concept of ordinal utility. However, if we are interested in inequality, the concept of ordinal utility becomes useless, for the cornerstone of the inequality concept is the assumption that the situation of individuals can be compared, not only in terms of better and worse, but also in terms of how much better or worse. If we want to compare individual well-being between individuals, it requires a cardinal well-being ${ }^{1}$ concept.

Notwithstanding this, the inequality concept has already a long history in economics. Things started with measuring income inequalities. These were statistical parameters that described the distribution of observed incomes. Well-known examples are the Pareto $\alpha$, the standard deviation of log-incomes or Theil's entropy measure. In terms of such measures perfect equality corresponds with a value zero.

Atkinson [1] was one of the first who devised an inequality measure that was more than a statistical measure. Although he never stated this explicitly, he proposed in fact that the relevant measure to gauge social inequality is not the inequality of incomes but the inequality of individual well-being. This inequality measure was based on a social welfare function (SWF) where minimum inequality corresponds with the situation where the social welfare function is maximized and due to concavity everybody enjoys an equal amount of utility or well-being. Atkinson suggested that this measure did not involve a cardinal utility concept, but this is debatable. Taking averages over utility implies a cardinality concept. The weakness of the approach was that there was no empirical foundation for the specification of the underlying utility function of income.

At about the same time (1971) the present author argued that by means of a subjective questioning technique one could define and estimate a cardinal welfare function of income, which later on became a key concept of the Leiden School literature and which can be seen as a forerunner of modern happiness economics estimation methods. By combining empirically estimated well-being with theoretical inequality indexes, the theoretical inequality concept could be empirically filled. A first example was how Atkinson's index was empirically implemented in Van Praag [23] (see also Van Praag and Ferrer-i-Carbonell [25], ch. 13)).

Apart from the cardinality issue there is a potential second problem with wellbeing inequality measures in general. Income inequality measures are explicitly or implicitly based on a transferable utility concept. Indeed, income can be redistributed. There are however more well-being determinants than income (e.g. health, age, education, and IQ). Most of these determinants cannot be redistributed but they are relevant for well-being, and inter-individual differences in those non-income determinants may cause feelings of well-being inequality as well. It follows that wellbeing inequality cannot be a simple generalization of income inequality, as feelings of inequality in well-being may be caused by many factors, only one of which is income. We do not have a simple ideal benchmark of what is minimal inequality either. Theoretically, this has to be equality of individual well-being, but this concept is empty, as long as we do not accept an empirical individual well-being concept.

\footnotetext{
${ }^{1}$ In line with the modern happiness economics literature we use the terms utility, welfare, wellbeing and satisfaction as standing for the same empirical concept. Although from a philosophical and semantic point of view we may differentiate between the concepts, in practice this appears difficult.
} 
Nowadays the results of happiness economics have led to a beginning acceptance of the possibility to estimate subjective well-being directly by means of evaluation questions of the type: how do you evaluate your life as a whole on a scale from 0 to 10, where zero stands for the worst and 10 for the best situation?

We refer to Frey and Stutzer [10], Layard [16], Van Praag and Ferrer-i-Carbonell [25, 27], Clark, Frijters, and Shields [4], Dolan et al. [5], Graham [11] for surveys of the blooming literature.

In this paper we shall argue that the present model used in happiness literature is essentially incomplete. The present literature ${ }^{2}$ is in essence about empirically estimating the equation $U=U(x)$, where $x$ stands for a vector of characteristics of the individual $x$. In relatively few contributions the impact of the reference group of the individual is recognized. This is done by including the average income $\bar{x}_{r e f}$ of the reference group and positing and estimating the extended model $U=U\left(x, \bar{x}_{r e f}\right)$. However, if we look at inequality this model is insufficient. Inequality summarizes the inter-individual comparison process, where both the question of how much importance the individual assigns to comparisons with others and the variation between individuals within the reference group plays an important role as well.

In Section 2 we shall discuss the operational concept of subjective well-being. In Section 3 we shall develop the corresponding well-being inequality concept. In Section 4 we shall take account of the fact that no individual evaluates in isolation, but that the circumstances of his reference group co-determine his norms on what is subjective well-being. It follows that the phenomenon of social transparency or lack of transparency plays a role in the evaluation of social subjective well-being. This calls for developing a model of the referencing process and the definition of a personal subjective inequality concept, which describes the inequality the individual perceives between his satisfaction level and the satisfaction levels of others in his reference group. In Section 5 we aggregate those feelings of personal inequality into a social subjective inequality concept.

The aim of this paper is to sketch a theoretical model of how the reference mechanism affects individual well-being and, consequently, the well-being inequality concept. Finally, we consider how these insights may contribute to the shaping of social policy. In this paper we do not give an empirical application. The reason for this is that we do not know of the existence of a data set that would make it possible to estimate the model. In Section 6 we discuss how these concepts might be made operational in practice.

In Section 7 we shall briefly consider the implications for social policy, while Section 8 concludes.

This paper may also be read as a plea for creating more empirical information on the referencing process by extracting information from individuals in surveys and experimental settings.

We hope that the model outlined in this paper may be a fruitful starting point for integrating the referencing mechanism in happiness economics. This is the final objective of this paper.

${ }^{2}$ In Section 2 we shall look more in detail on the present literature. 


\section{Subjective well-being}

The concept of subjective well-being is empirically based on the so-called satisfaction questions like the one cited in the introduction. Such satisfaction questions can also be posed with respect to life domains, such as health, financial situation and job situation, yielding empirical evaluations of domain satisfaction or domain well-being. The answers to such questions are mainly categorized on a numerical scale from 0 to 10,1 to 10 , or 1 to 7 . There is now a growing consensus that such answers have cardinal significance [6]. Respondents have a conception of a worst and a best situation and they situate their situation between those points. At the moment nearly all empirically used question modules are categorized, such that only the points 0,1 , $2, \ldots$, and 10 are possible answers, but it does not need much fantasy to assume that in the near future those answers will be asked and given on a continuous scale by the respondent who positions himself on a continuous line segment where the left end-point stands for the evaluation of the worst conceivable situation and the right end-point for the best conceivable situation. Let the situation itself be described by some characteristics like 'income', 'health status', 'age', in short a vector $x$; then the evaluation of $x$ is described by a number $U(x)$. We will call such a function a satisfaction function. ${ }^{3}$

The most simple approach is now to denote the responses on the satisfaction question by $U$, which can assume values $0,1,2, \ldots$, and 10 , and to postulate an explanatory model like

$$
U_{n}=\beta_{0}+\beta_{1} \text {. income }_{n}+\beta_{2} . \text { age }_{n}+\beta_{3} \text {. } \text { familysize }_{n}+\varepsilon_{n}
$$

where the variable income stands either for household income of for its logarithm. The other variables are also defined either by absolute values or by their logarithms. This specification or similar ones yield very interesting and stable results (see e.g. [2]).

The problem with this specification is that the RHS can assume values outside the range $[0,10]$. An easy transformation avoids this logical problem.

We may describe $U(x)$ by a tractable distribution function on $(-\infty,+\infty)$ with the range $[0,1]$. This suggests the normal or the logistic as obvious choices. We assume

$$
U_{n}=N\left(\beta_{0}+\beta_{1} \text {.income }_{n}+\beta_{2} . \text { age }_{n}+\beta_{3} \text {. } \text { familysize }_{n} ; 0,1\right)
$$

where $N(. ; 0,1)$ stands for the standard normal distribution function.

Denoting the inverse by $u_{n}=N^{-1}\left(U_{n}\right)$ and adding an error term we get the OLS model

$$
u_{n}=\beta_{0}+\beta_{1} \cdot \text { income }_{n}+\beta_{2} \cdot \text { age }_{n}+\beta_{3} \text {. familysize } \text { fa }_{n}+\varepsilon_{n}
$$

It has been empirically found (see e.g. [25], and also [8] for related results) that the estimated trade-off ratios $\beta_{i} / \beta_{j}$ for both specifications are nearly always not statistically different from each other. Actually, this is not that strange as both formulations are describing the same indifference curves on the (income, age, familysize)-space. They are just two different cardinalizations of the same preference ordering.

\footnotetext{
${ }^{3} \mathrm{We}$ avoid the term utility, since in all empirical questions the word 'satisfied' is used.
} 
These satisfaction functions are subjective and individualized. They are subjective, because they are derived from gauging subjective feelings. They are individualized because individual variables determine life satisfaction.

\section{Subjective inequality}

Up to now there are only a few attempts to define inequality with respect to happiness or subjective well-being ${ }^{4}$ (SWB) $[9,25]$ ). Nevertheless, the same need for income inequality definitions that has produced such a flourishing literature in economics is now even more strongly felt with respect to the concept of happiness or satisfaction inequality. If we are looking for a definition of subjective inequality it should be based on these measured subjective satisfaction functions $U(x)$.

Here, the basic ingredient is the observed response $U$ and one of the corresponding happiness Eqs. (2.1), (2.2) and (2.3). If all individuals in our sample would enjoy the same SWB-level $U$, it would imply that SWB-inequality is minimal. This situation may occur even if the SWB-determinants income, age and family size are different between individuals. The only thing that counts is that their corresponding $U$-values according to (2.3) are identical. The individuals have to be on the same indifference curve. The advantage of this definition on the basis of subjective, individually specified, satisfaction functions is that satisfaction or well-being is not only determined by income but by many other determinants as well, such as age, number of children, and health. For instance, there is strong evidence that the age and health of the individual are determinants of life satisfaction. It follows then that part of the observed inequality in well-being may be explained by differences in age and health. If individuals have the same $U$ (or $u$ )-value, they enjoy the same level of subjective well-being. Moreover, the individualization implies that different individuals may evaluate the same objective situation (e.g. characterized by income) differently, depending on personal characteristics such as age and health.

Now we may define inequality with respect to SWB in a similar manner as it has been defined with respect to income. That is, any income inequality index $I\left(y_{1}, \ldots, y_{N}\right)$ has his SWB-analogue $I\left(u_{1}, \ldots, u_{N}\right)$, The $u$-variable takes over the role of (log-)income. Surely, definition of a SWB- inequality index implies accepting the cardinal significance of $U$, as the measurement of inequality implies giving a meaning to the difference between various levels of SWB. Consequently, if we do not believe in a cardinal significance of the responses to satisfaction or happiness questions, then it is impossible to define an SWB-inequality index, irrespective of the specific definition of that inequality index $I\left(u_{1}, \ldots, u_{N}\right)$. This is true, notwithstanding the fact that under an ordinal interpretation complete equality may be identified as the situation, where all individuals assign an equal satisfaction value $U$ to their situation.

In this paper we choose for a rather simple and intuitive definition of SWBinequality. We specify inequality by the variance (or standard deviation) of $u$ over the sample or population. As already said, we may also use any other usual income

\footnotetext{
${ }^{4}$ Notice that happiness inequality is something else as the effect of income inequality on individual well-being. We refer to Graham and Felton [12] for an interesting study on this latter relation for Latin-American countries.
} 
inequality measure like the Gini-index, the Theil-index, Pareto- or the Atkinsonindex. However, in this context, where we aim at introducing the reference group effect within an inequality context, we take the inequality definition which is most convenient for the exposition.

Let us assume (2.3), that is

$$
u_{n}=\beta_{0}+\beta_{1} \text {. income }_{n}+\beta_{2} . \text { age }_{n}+\beta_{3} \text {. familysize } \text { fam }_{n}+\varepsilon_{n},
$$

or more generally

$$
u_{n}=\beta_{0}+\sum_{i=1}^{k} \beta_{i} x_{i n} .
$$

where we ignore the random error. The nice consequence of the existence of a relationship like (3.2) is that we may trace the effects of changes in personal characteristics $x_{n}=\left(x_{1 n}, \ldots, x_{k n}\right)$ on overall well-being.

The first approach according to which we might define subjective well-being inequality with respect to a specific population is

$$
\sigma^{2}(u)=\beta^{\prime} \Sigma_{x x} \beta
$$

Here the variance is taken with respect to the population density $f(x)$ of the vector of relevant characteristics $x$, and $\Sigma_{x x}$ is the population covariance matrix of the vector $x$ of welfare determinants. The log-income variance is one of its diagonal elements. If SWB is only dependent on log-income, it is obvious that SWB-inequality is just $\beta^{2} \sigma^{2}$ $(\log (y))$. If we assume the implicit classical assumption that SWB equals log-income, then $\beta=1$, and we end up with the traditional variance of log-incomes.

We see here two points to be noticed. First, the vector $\beta$ makes the variance subjective. The vector $\beta$ is assessed on the basis of subjective questions on how satisfied individuals are. Differences with respect to variables that correspond to a relatively large $\beta$ and consequently have a sizeable impact on individual well-being will have a strong influence on overall variance, while variables with a relatively small $\beta$ will have a small effect on overall variance as well. If income is included as one of the variables $x$, then income inequality is one of the components of SWB-inequality, but inequality in other variables have influence on $u$ or well-being $U$ as well. Second, we see that (3.3) depends on the population covariance matrix $\Sigma_{x x}$. Hence, $\sigma^{2}(u)$ may be seen as an aggregate of population inequalities with respect to the various component variables $x_{i}$, corrected for possible correlations between them. It is also possible to assess the effect of changes in the $x$-distribution. For instance, if $x_{1}$ stands for log-income, then a change in the variance of log-income $\sigma_{11}$ or its covariance $\sigma_{12}$ with another variable $x_{2}$ will immediately change the overall inequality index. This index may be seen as a tool for making government policy. In the hypothetical case that there is no objective inequality with respect to the relevant determinants $x$, that is $\Sigma_{x x}=\mathbf{O}$, subjective inequality will be zero as well.

If the inequality index is a political tool, then we may ask which variables $x$ are considered as being relevant by the government. For instance, is the number of children relevant for making government policy? If we should not think so, we have to ignore the SWB-differences due to the children effect, although it is intuitively obvious that family size inequality affects life satisfaction inequality. It means that we have to re-estimate (3.1) without including the variable family size. If that variable 
is correlated with other explanatory variables, as it most probably is, it will imply that the estimate of the vector $\beta$ will change as well. This shows that the choice of explanatory variables is rather relevant for the definition and the measurement of subjective inequality, and this makes the choice of which variables are considered to be relevant for including in the inequality definition a political matter as such.

\section{Personal subjective inequality as felt by individuals within reference groups}

The inequality index just considered does not take into account the reference phenomenon. Does this index account properly for the impact of the refereeing process on the feelings of inequality of individual citizens? Probably it does not. The reason is that the evaluation by individuals of their own situation is partly done by comparing their own situation with that of others, the so-called reference group.

It has been found by several authors $[7,13,15,17,19-22,26]$ that satisfaction with life or with one's financial situation depends not only on own income $y_{n}$ but on the average income of the reference group of $n$, say $\overline{y_{n, r e f}}$, as well. For instance, (3.1) carries over into

$$
u_{n}=\beta_{0}+\beta_{1} \text {.income }_{n}+\beta_{2} \cdot \text { age }_{n}+\beta_{3} . \text { familysize }_{n}+\gamma \cdot \overline{y_{n, r e f}}+\varepsilon_{n} .
$$

The effect of own income is positive. The age effect is regularly found to be parabolic, first decreasing and after about 40 increasing (see e.g. [2, 18]; in [27] even a cubic age curve is proposed). For reasons of exposition we ignore the squared term. The effect of children on life satisfaction is ambiguous. The effect of reference income is mainly estimated to be negative, that is, own satisfaction reduces if neighbors get more, although some authors like Senik [19] found a positive effect, e.g., for ex-Soviet citizens.

Actually, the problem is how to describe the reference group. Mostly this is intuitively defined by assumption. The reference group is equated to persons belonging to the same age bracket, education group, region, etc. However, this a priori definition discards the possibility that we can learn from the data what the composition of the reference group really is.

In order to get a more detailed description of the reference group and its influence we need to look more in detail. We shall outline the idea by a simple example. The group consists of various reference individuals with whom the individual in question, called Peter, compares himself. Say, Peter's reference group consists of John and Adam. Now we assume that Peter is not always busy with comparing his situation to that of others. Sometimes he is self-oriented and sometimes other-oriented. Let us assume he is self-oriented for $60 \%$ of his time. For $30 \%$ of the remaining time he is oriented on John and for $10 \%$ on Adam. Obviously John is the more important reference person for him. Let us now assume that the incomes of the three persons are ordered as $y_{J}>y_{P}>y_{A}$. Then it seems reasonable to assume that the individual feels less happy if he has John in mind, than when he has Adam in mind, while his situation is in the middle when he is self-orienting. Actually, it is only one step to assume that $y_{n, r e f}$ is a random variable itself and that consequently perceived wellbeing $u_{n}$ is random as well. Well-being varies with the reference person or social type we happen to have in mind to compare with. Equation 4.1 is just the expectation of $u$ over the reference group. 
For convenience, let us assume that the only relevant reference characteristic is income and that income is measured bracket-wise. The bracket averages are $y_{r e f, 1}, \ldots$, $y_{r e f, k}$. The individual compares sometimes with people in the first bracket, say a fraction $p_{1, n}$ of the time, sometimes with people in the second bracket, say a fraction $p_{2, n}$ of the time, and so on. Those individuals may be seen as representing social types $\kappa=1,, k$.

Then we may write (4.1) more explicitly as

$$
u_{n}=\beta_{0}+\beta_{1} . \text { income }_{n}+\beta_{2} . \text { age }_{n}+\beta_{3} \text {. familysize } \text { far }_{n}+\gamma\left[p_{1, n} y_{r e f, 1}+\ldots+p_{k, n} y_{r e f, k}\right]+\varepsilon_{n} .
$$

Here each income bracket $\kappa$ is weighted by the importance it has in the reference group of individual $\mathrm{n}$. The weights, which add up to one, are denoted by $p_{1, n}, \ldots, p_{k, n}$. The corresponding distribution we may call the reference distribution. It may be that every individual has the same reference group weights $p_{1, n}, \ldots, p_{k, n}$ but it is more probable that different individuals will have different reference distributions. If all reference distributions are the same, the weights $p_{1, n}, \ldots, p_{k, n}$ would probably reflect the objective population fractions $p_{1}, \ldots, p_{k}$ of the different income brackets. If not, the fractions $p_{i, n} / p_{i}$ may be larger or smaller than one. If $p_{i, n} / p_{i}>1$ it follows for $n$ that he overweighs the importance of people in bracket $i$, while $p_{i, n} / p_{i}<1$ implies the opposite. This ratio $p_{i, n} / p_{i}$ was termed in earlier work (see [25] ch.8 and earlier in [24]) the social filter, through which individual $n$ perceives the society around him.

We may generalize this idea to a continuous reference group where the referencing characteristic $Y_{r e f}$ may take any value on the real axis. Then the reference distribution is described by a density function $\underline{f}_{\text {ref,n }}(y)$ and the average reference income to be included in (4.2) would become $\bar{Y}_{r e f}=\int y \cdot f_{r e f, n}(y) d y$. It is obvious now that apart from comparing our own income with reference income we may also compare our age to reference age, and our family size to reference family size. If those variables are determinants for happiness, it may be surmised that the same variables of the reference persons may have an impact on our happiness as well. The same holds for other variables held to be relevant. In short, the reference variable may be more-dimensional vector. Then it follows that we may define a random reference vector $X_{r e f}=\left(Y_{r e f}, A g e_{r e f}, F_{a m s_{r e f}}\right)$ with a corresponding more-dimensional reference density function $f_{r e f, n}(x)$ and an average vector $\bar{X}_{r e f}$. Similarly we may define a variance covariance-matrix with respect to the reference distribution, denoted by $\Sigma_{r e f}$

Let us now generalize (4.1) in this vein. We start simply by assuming a two-person world where an individual, say Peter, has one reference person, let us call him John, to compare with. Peter's situation is described by the vector $X_{P}$, and John's situation by $X_{J}$.

Even in this very simple two-person world Peter may have a perception of inequality of SWB, when he compares his situation with that of John. As already said, an individual is not comparing all the time. More precisely, if an individual is never comparing with his neighbor, he will not perceive inequality at all. If he compares at times, then his well-being is determined by an absolute component $X_{P}$ and by a relative component $\left(X_{P}-X_{J}\right)$, the difference between Peter's and John's situation. We assume that people compare their situation with others only for a fraction $(1-\pi)$ of their time while for a fraction $\pi$ they look only at their own situation without comparing. We call the fraction $\pi$ the self-orientation coefficient of the individual. Its 
complement $(1-\pi)$ may be termed the outwards-orientation coefficient. It follows that we assume that satisfaction $u_{P}$ is not constant for an individual but that it depends on his or her mood of the moment, whether he or she is comparing or noncomparing his situation with that of others. In short, we assume that $u_{P}$ is a random variable, defined as

$$
u_{p}= \begin{cases}\beta_{0}+\beta X_{P} & \text { with chance } \pi \\ \gamma_{0}+\gamma\left(X_{P}-X_{J}\right) & \text { with chance }(1-\pi) .\end{cases}
$$

Life satisfaction, even during a relatively short period, is not constant but it is random, depending on whom one is comparing to at the moment. We might call it instantaneous satisfaction. The corresponding cardinal value on $[0,1]$ is found by means of (2.2).

In the first situation in (4.3) Peter looks only at his own situation, he is selforiented. In the second situation it is only the difference between him and John that counts. Notice that in this simple specification even if Peter and John are in the same situation, this does not imply that the individual ' $\mathrm{s} u_{P}$ is the same in both situations. Just the fact that both are felt to be in the same situation as such may make Peter feel less happy or more happy. It is evident that this specification is just an example.

We notice that the expectation $E\left(u_{P}\right)$, like in (4.2), is a linear function in $X_{P}$ and $X_{J}$

$$
E\left(u_{P}\right)=\pi \cdot \beta_{0}+(1-\pi) \gamma_{0}+\pi \cdot \beta X_{P}+(1-\pi) \gamma\left(X_{P}-X_{J}\right) .
$$

If the true model is (4.4), where $y_{n, r e f}$ is a random variable depending on whether we compare or not at the moment, we are in fact estimating its expectation (4.1).

We notice that the parameter $\pi$ is unknown and has to be estimated as well. We cannot empirically identify $\beta$ without further information with respect to the comparison chance. Perhaps, the Day Reconstruction Method, as described by Kahneman et al. [14], can shed light on what is the frequency of comparison moments.

Now we may also define a feeling of personal well-being inequality as felt by Peter. It is

$$
\begin{aligned}
\sigma^{2}\left(u_{P}\right) & =\pi\left[\beta_{0}+\beta X_{P}-E\left(u_{P}\right)\right]^{2}+(1-\pi)\left[\gamma_{0}+\gamma\left(X_{P}-X_{J}\right)-E\left(u_{P}\right)\right]^{2} \\
& =\pi(1-\pi)\left[\beta_{0}-\gamma_{0}+\beta X_{P}-\gamma\left(X_{P}-X_{J}\right)\right]^{2}
\end{aligned}
$$

We see that the feeling of inequality is zero, if the self-orientation $\pi$ is either zero or one. The feeling of inequality is the highest, if Peter is self-oriented half of the time, that is $\pi=1 / 2$. It is also evident that Peter and John may have a different perception of the inequality between them as their $\pi$ 's may be unequal and/or their satisfaction functions may differ.

A generalization of this definition using other specifications of the satisfaction index $u_{P}$ than in (4.3) lies at hand.

Let us now extend the concept of a reference group from a one-person group to a multi-person group. Each social type is characterized by a vector $X$. Now instantaneous satisfaction is defined, analogously to (4.3), as

$$
u_{n}=\left\{\begin{array}{l}
\beta_{0}+\beta_{1} \text {.income }_{n}+\beta_{2} . \text { age }_{n}+\beta_{3} . \text { familysize }_{n} \quad \text { with chance } \pi \\
\left(=\beta_{0}+\beta^{\prime} X_{n}\right) \\
=\gamma_{0}+\gamma^{\prime}\left(X_{n}-X_{\text {ref,n }}\right) \quad \text { with chance } 1-\pi .
\end{array}\right.
$$


The vector $X_{r e f, n}$ is random and drawn from $n$ 's reference distribution with density function $f_{r e f, n}(x)$. We have here a random choice process in two stages. The first choice determines whether the individual is in a comparing or a non-comparing mood, chances being $(1-\pi)$ and $\pi$, respectively. Second, the question is which specific reference type $X_{r e f, n}$ is coming into $n$ 's mind, when he is actually comparing. This is described by the reference density function $f_{r e f, n}(x)$. Since more persons may be described by the same vector $x_{r e f, n}$, it is the description of a social type. The random vector $X_{r e f, n}$ is varying over the space of social types. Notice that the expectation of $u_{n}$ is

$$
E\left(u_{n}\right)=\mu_{n}=\pi \cdot\left[\beta_{0}+\beta^{\prime} X_{n}\right]+(1-\pi)\left[\gamma_{0}+\gamma^{\prime}\left(X_{n}-\bar{X}_{r e f, n}\right)\right]
$$

where $\bar{X}_{r e f, n}$ is the vector of expectations of $X_{r e f, n}$ with respect to the reference density function $f_{r e f, n}(x)$. One may call the average vector $\bar{X}_{r e f, n}$ the social focal point of $n$. Notice that it is by no means necessary that $\bar{X}_{r e f, n}=X_{n}$, that is, that individuals take their own type as the focal point of their reference group. More usually they may take somebody or some social type, who is socially above them, as a social focal point. Equation (4.7) or rather the expectation of (4.6) with respect to $X_{r e f, n}$ is estimated in the happiness literature. It is linear in $o w n$ characteristics and in the average characteristics $\bar{X}_{r e f, n}$ of the reference group. Up to now in the empirical literature reference groups are defined in terms of income only. This implies that all elements of the parameter vector $\gamma$ are assumed to be zero, except the element $\gamma_{y}$, which refers to income. There is however nothing against it to characterize reference persons by a more-dimensional vector of characteristics instead of one-dimensionally by income only.

In accordance with the definition in (4.5) in the case of a multi-person reference group we now define the feeling of personal subjective inequality from the viewpoint of individual $n$ as

$$
\sigma^{2}\left(u_{n}\right)=\pi(1-\pi)\left[\beta_{0}-\gamma_{0}+\beta^{\prime} X_{n}-\gamma^{\prime}\left(X_{n}-\bar{X}_{r e f, n}\right)\right]^{2}+(1-\pi) \cdot \gamma^{\prime} \Sigma_{r e f, n} \gamma
$$

This formula is based on the well-known variance decomposition formula. There are now two 'subgroups' distinguished. The first is the one-person group consisting of the individual $n$ himself, while the second subgroup is his or her reference group, consisting of many different social types. Hence, there is a 'between'-inequality described by the first term in (4.8) and a 'within'-inequality of the reference group itself, given by the second term.

Personal subjective inequality appears to depend on four elements. First, it depends on the self-orientation coefficient $\pi$; second, it depends on the perceived difference between the individual and his or her reference group; third, on the vector $\gamma$, that is, on the effects $\gamma$ of the reference vector elements on satisfaction. Some components have strong influence like reference income, while others presumably will have negligible influence; fourth, it depends on the spread within the reference group, that is, the covariance matrix $\Sigma_{r e f, n}$. It describes in a sense the individual's horizon of society as perceived by individual $n$. If one diagonal element, say corresponding to reference income, is larger than another, say, with respect to reference age, it means that the reference group of $n$ is wider with respect to income than with respect to age. 
We may interpret the off-diagonal elements, say $\sigma_{r e f, i j}$, in a similar way. If the covariance (or the correlation) is strongly positive it implies a strong positive correlation within the reference group between, for example, income and age. A negative correlation may be interpreted likewise.

It is obvious that the reference covariance matrix $\Sigma_{r e f, n}$ is related to but not necessarily identical with that of the objective population distribution. More specifically, if the underlying population is perfectly homogeneous with respect to a characteristic $X_{i}\left(X_{r e f, i}\right.$ is constant) and hence the corresponding population variance $\sigma_{i i}=0$, the reference group mostly will be perceived as perfectly homogeneous as well with respect to that characteristic $X_{i}$, that is, $\sigma_{r e f, i i}=0$ as well. It follows then that the corresponding population and reference covariances are zero as well.

We notice that all parameters seem to be estimable, when we are able to get more specific observations on the individual reference process. We need answers to the question of how frequently an individual compares his own situation with that of others, yielding a $\pi$-estimate and we need to know with whom the individual compares to get an idea of the reference density function $f_{\text {ref,n }}(x)$. That is, we need per individual $n$ with characteristics $X_{n}$ a sample $\left\{X_{r e f, n, m}\right\}_{m=1}^{M}$ of order $M$ in order to estimate $n$ 's reference distribution.

\section{Social subjective inequality with a referencing mechanism}

Policy makers are not so much interested in the inequality feelings of one person, as specified in by (4.8) by $\sigma^{2}\left(u_{n}\right)$, but rather in the average feeling of social inequality in society at large, that is $E_{n}\left[\sigma^{2}\left(u_{n}\right)\right]$, where the average is taken with respect to all members $n$ of the population.

Now we have to account for the fact that each person may have his or her own reference group. For each person $n$ we define again the vector of satisfaction determinants $\left(X_{n}, X_{r e f, n}\right)$. Its dimension is $2 k$. We call the first half the objective determinants. They describe the situation of person $n$. The second half of the vector stands for characteristics of reference persons of $n$. They are called the reference values. We can ask the person $n$ at a specific moment in time with whom or what social type he is comparing himself. The answer is $X_{r e f, n}$. The first vector is pretty fixed per person, but $X_{r e f, n}$ may vary per moment. It is random. Its distribution is the reference distribution of $n$.

If we consider the whole population, then $\left(X_{n}, X_{r e f, n}\right)$ may be considered as a random vector defined on the whole population with an expectation $\left(\bar{X}, \bar{X}_{r e f}\right)$ and a $(2 k \times 2 k)$ - covariance matrix

$$
\Sigma=\left(\begin{array}{cc}
\Sigma_{x x} & \Sigma_{x x_{r e f}} \\
\Sigma_{x_{r e f}} & \Sigma_{x_{r e f} x_{r e f}}
\end{array}\right)
$$

Now it is tempting to assume the distribution to be normal, which may be realized after suitable redefinition of the variables. For example, taking logs frequently helps a good deal. Still better is to apply an integral transformation where quantiles of the empirical distribution function are mapped on the corresponding quantiles of the standard normal distribution function. Again, we observe that choosing for 
normality after suitable transformation of the observations does not imply that the model cannot be generalized to non-normal distributions. However, for the sake of exposition we assume normality. In fact, whether a normal specification is realistic, has to be inferred from empirical observation.

Now it appears possible and appropriate to assume that different individuals have different reference groups, and, consequently, that they have different reference distributions. We see that the individual reference distributions, introduced before, may be interpreted as conditional distributions of $X_{\text {ref }}$, given the objective determinants $X$ of $n$. A very pleasant property of normal distributions is that the conditional distributions have different means, but the same constant covariance matrix.

The conditional averages of those individual reference distributions will vary with the objective characteristics $X_{n}$. For a multivariate normal distribution we have

$$
\bar{X}_{r e f, n}=E\left(X_{r e f, n} \mid X_{n}=x_{n}\right)=\bar{X}_{r e f}+\Sigma_{x_{r e f} x_{r e f}}^{-1} \Sigma_{x_{r e f} x}\left(x_{n}-\bar{X}\right) .
$$

As before, we call this conditional average the social focal point of $n$. The focal point varies with the objective characteristics $x_{n}$. Mostly we may assume a positive correlation between objective individual characteristics $x_{n}$ and reference characteristics, as individuals tend to compare themselves with those who belong to the same social group. If $\Sigma_{x x_{\text {ref }}}=O$, the gravity point of the reference distribution is constant. In a similar way using the well-known formulae of normal distribution theory we find that the covariance matrix of the conditional distribution of $X_{r e f, n}$ is

$$
\Sigma_{r e f, n}=V\left(X_{r e f, n} \mid X_{n}=x_{n}\right)=\Sigma_{x_{r e f} x_{r e f}}-\Sigma_{x x_{r e f}} \Sigma_{x x}^{-1} \Sigma_{x_{r e f} x} .
$$

This conditional covariance matrix is the same matrix $\Sigma_{r e f, n}$ which we used in (4.8). It is obviously smaller (in the sense of matrix ordering) than the overall reference covariance matrix $\Sigma_{x_{\text {ref }} x_{\text {ref }}}$. Under the assumption of normality this matrix is constant, that is, $\Sigma_{r e f, n}=\Sigma_{r e f}$.

It follows from (5.2) that

$$
\begin{aligned}
X_{n}-\bar{X}_{r e f, n} & =X_{n}-\bar{X}_{r e f}-\Sigma_{x_{r e f} x_{r e f}}^{-1} \Sigma_{x_{r e f} x}\left(X_{n}-\bar{X}\right) \\
& =C+(I-B) X_{n}
\end{aligned}
$$

where $C$ is a constant vector and $B$ is the matrix of 'regression' coefficients of $\bar{X}_{r e f, n}$ on $X_{n}$.

Now we may define overall social inequality as the expectation of (4.8) over the population.

Using (5.4) we rewrite

$$
\begin{aligned}
\beta_{0}-\gamma_{0}+\beta^{\prime} X_{n}-\gamma^{\prime}\left(X_{n}-\bar{X}_{r e f, n}\right) & =\beta_{0}-\gamma_{0}-\gamma^{\prime} C+\left(\beta^{\prime}-\gamma^{\prime}(I-B)\right) X_{n} \\
& =\tilde{C}+\tilde{D}^{\prime} X_{n}
\end{aligned}
$$

where $\beta_{0}-\gamma_{0}-\gamma^{\prime} C=\tilde{C}$ is a scalar and where $\left(\beta^{\prime}-\gamma^{\prime}(I-B)\right)=\tilde{D}^{\prime}$ is a row vector. 
It follows then that

$$
\begin{aligned}
E_{n}\left[\sigma^{2}\left(u_{n}\right)\right] & =E_{n}\left[\pi(1-\pi)\left[\beta_{0}-\gamma_{0}+\beta X_{n}-\gamma^{\prime}\left(X_{n}-\bar{X}_{r e f, n}\right)\right]^{2}+(1-\pi) \cdot \gamma^{\prime} \Sigma_{r e f, n} \gamma\right]= \\
& =E_{n}\left[\pi(1-\pi)\left[\tilde{C}+\tilde{D}^{\prime} X_{n}\right]^{2}+(1-\pi) \cdot \gamma^{\prime} \Sigma_{r e f, n} \gamma\right]= \\
& =\pi(1-\pi)\left[(\tilde{C})^{2}+2 \cdot \tilde{C}\left(\tilde{D}^{\prime} \bar{X}_{n}\right)+\tilde{D}^{\prime} \Sigma_{x x} \tilde{D}\right]+(1-\pi) \cdot \gamma^{\prime} \Sigma_{r e f} \gamma .
\end{aligned}
$$

If $\pi=1$, that is no comparison with other individuals, the inequality is zero. In the case that the individual derives his satisfaction completely by comparison $(\pi=0)$ the inequality will be $\gamma^{\prime} \Sigma_{x x} \gamma$.

It follows that subjective inequality does not only depend on the inequality with respect to own welfare determinants, but that it also depends on the individual reference effects and on the inequality in the reference group.

In the above we made the convenient assumption that the underlying distribution of $\left(X_{n}, X_{r e f, n}\right)$ would be multivariate normal. Although not unreasonable as a first approximation, it is not really needed. The conceptual model just described holds for any distribution, but only the formulae become less elegant or have to be replaced by numerical calculations.

\section{Where to find the data?}

Unfortunately the model is not yet operational by lack of data. The problem is obviously how to estimate the matrices $\Sigma_{x x_{r e f}}, \Sigma_{x_{r e f} x_{r e f}}$ and the comparison chance $\pi$. This is only possible if we can observe per individual the whole vector $\left(X_{n}, X_{r e f, n}\right)$ and not only the first half of that vector. This implies repeated interviewing in order to get an idea per individual of his/her reference distribution and to get an estimate of the self-orientation parameter $\pi$, that is how frequently he compares with other people. A relatively rare example of frequent interviewing is the diary method, as applied by Kahneman et al. [14], which they call the Day Reconstruction Method.

A first inspiration content-wise is given by a recent paper by Clark and Senik [3] who analyzed two questions put in Wave 3 of the European Social Survey (ESS). One question is the following: "Whose income would you be most likely to compare your own with? Please choose one of the groups on this card: Work colleagues/Family members/Friends/Others/Don't compare/Not applicable/Don't know." This question, which was very well responded to, is in the spirit of the questions we would have in mind.

We would suggest for comparisons to construct a $X_{r e f, n}$-vector some nuclear questions like these:

Everybody compares his situation with that of others. When you compare yourself other persons, take the person (perhaps plural? To make the respondent think about a group of people) in mind with whom you most frequently compare. Call him John/Betsy. Please, can you describe some features of Betsy

1. What might be Betsy's net household income about?

2. How would you classify Betsy's health on a scale from 0 to 10 ? 
3. How old is Betsy?

4. What is her family size?

5. Her age?

6. Her employment status?

7. Her education?

8. Would you classify her in the first place as work colleague/family member/friend/others/not applicable/don't know

9. How often do you see Betsy?

10. Do you think that Betsy is happier, equally happy, or less happy in life than you?

11. Would you be happier, equally happy, or less happy in life when you were Betsy than you are feeling now being yourself?

It is obvious that this is just a first suggestion on possible questions, but it is clear that repeated interviews would give the researcher an idea about who are the reference persons of each individual, how they look like, and concretely, about the distribution of $X_{r e f, n}$. As there is frequently more than one reference person, we could try to ask the same question for a second person, say Peter. Moreover, we could try to get an idea of how frequently the respondent compares with Betsy and how frequently with Peter. Obviously this can be extended to more reference persons. It is in this way that we think that the reference group model outlined above may be operationalized.

\section{What does this mean for social policy?}

Let us assume a government which is interested in enhancing the well-being of its people. It formulates a Social Welfare Function (SWF) analogous to Markowitz' portfolio theory, as

$$
\mathrm{SWF}=\delta \cdot E(u)-(1-\delta) \cdot \sigma^{2}(u) .
$$

It is a $\delta$-weighted average of average individual SWB and inequality in individual SWB, where social inequality is negatively signed, assumed to be bad. The SWF has to be maximized and the question is now what instruments the government can use. Obviously, there are some objective characteristics $X$ like e.g. income, education, and health which may be influenced by government, although not without a cost attached. A change in the parameters $\beta$ will be rather difficult as they describe real preferences. The same holds more or less for the parameters $\gamma$. They stand for the jealousy effects, which seem to be fixed elements of human nature and although we do not deny that ethical/religious indoctrination may reduce jealousy effects, we do not think that this is a very powerful policy nowadays.

However, a final element is the matrix $\Sigma_{x_{r e f} x_{r e f}}$. It describes the transparency of society, and there holds the larger the transparency, the greater personal inequality feelings will be.

We may write (7.1) more explicitly as

$$
\mathrm{SWF}=\delta \cdot\left(\left(\pi\left(\beta_{0}+\beta^{\prime} \mu_{x}\right)+\gamma^{\prime}(1-\pi)\left(\bar{X}-\bar{X}_{r e f}\right)\right)+-(1-\delta) \cdot\left(\sigma^{2}(u)\right) .\right.
$$

The second term may be written more explicitly by substituting (5.5). From this formula (7.2) it is clear that the parameters of the referencing mechanism play an important role in the perception of the SWF. As we saw already, the reference 
distribution is a description of how visible other people are for a citizen. The covariance matrix describes the social transparency of society with respect to a number of relevant dimensions, described by the dimensions of $X$.

It is obvious that the referencing process is a sociological phenomenon, which can be influenced. We think here especially of media policy. For instance, when television disseminates on a day to day basis how the rich are living it is obvious that the frequency of comparing and social transparency is increased enormously. In formula it would imply that $\Sigma_{x_{\text {ref }} x_{r e f}}$ is increased while $\pi$ and $\Sigma_{x x_{r e f}}$ are decreased, as social segmentation is reduced.

The same holds on a global level for global inequality feelings. Hence, governments, and to a lesser extent publications in radio and printed journals and newspapers, have a non-negligible and perhaps even enormous effect on the referencing mechanism. Although it is fashionable to welcome more social transparency, it is a matter to be discussed whether this tendency is good from a standpoint of social well-being. The model outlined above suggests that there are risks involved.

There will be costs associated with the manipulation of $\pi, \bar{X}_{r e f}, \Sigma_{x x_{r e f}}, \Sigma_{x_{r e f} x_{r e f}}$. These costs may be purely monetary but they may also be of a non-monetary nature. For instance, a reduction of social transparency will be considered by some as a loss, especially by the press. If we try to maximize the SWF we may add a social cost-function of the type $C\left(\pi, \bar{X}_{r e f}, \Sigma_{x x_{r e f}}, \Sigma_{x_{r e f} x_{r e f}}\right)$, and find an optimal social transparency by setting

$$
\begin{aligned}
\frac{\partial S W F}{\partial \pi}-\frac{\partial C}{\partial \pi} & =0 \\
\frac{\partial S W F}{\partial \Sigma_{x x_{r e f}}}-\frac{\partial C}{\partial \Sigma_{x x_{r e f}}} & =0 \\
\frac{\partial S W F}{\partial \Sigma_{x_{r e f} x_{r e f}}}-\frac{\partial C}{\partial \Sigma_{x_{r e f} x_{r e f}}} & =0 \\
\frac{\partial S W F}{\partial \pi}-\frac{\partial C}{\partial \pi} & =0 .
\end{aligned}
$$

\section{Conclusion}

In this paper we built a model to include the social referencing mechanism into happiness economics. We do not have the illusion that this model will be estimable in a year from now. However, as Section 6 suggests, it is certainly conceivable to estimate the missing parameters of the reference mechanism by posing suitable questions.

It is well-known that comparing with reference groups affects feelings of individual subjective well-being. In this paper we argue that referencing affects inequality feelings as well. The subjective inequality concept was introduced in Van Praag and Ferrer-i-Carbonell [25]. When we are investigating inequality of subjective well-being we have to recognize that interpersonal comparisons by individuals with their reference persons must be at the basis of the inequality concept. Therefore we defined an individual's reference group as a distribution of reference persons 
described in terms of the same characteristics which we deem to be relevant satisfaction determinants for the individual himself.

If we assume that the individual's well-being is partly determined by comparison with his reference group, the same will hold for his perception of the inequality of well being; in fact, it is the well-being inequality within his reference group plus the inequality between the individual and his reference group as a whole.

Then we make a distinction between the individual's SWB-inequality as perceived by individuals and social SWB-inequality, which is an average of the individual subjective well-being inequality perceptions.

If we assume that social well being, as described by a social welfare function, depends on individual subjective well-being and on the individual's perception of SWB-inequality, it follows that the reference mechanism as such may have an effect on the social welfare function. If a government accepts the task of increasing SWB, it may see influencing the social reference mechanism as a legitimate policy instrument.

The present paper is a first and necessarily mainly theoretical exercise on this line. At the moment we do not know of available data to operationalize our model empirically. However, we outline how, as an extension of existing questionnaires, we may add new questions by means of which it becomes empirically possible to estimate the parameters of the referencing mechanism in practice. If such data are realized, the way lies open for an empirical operationalization of this model.

We hope by this paper to have drawn more attention to the probably important role of the referencing mechanism for the implementation of social policy.

Open Access This article is distributed under the terms of the Creative Commons Attribution Noncommercial License which permits any noncommercial use, distribution, and reproduction in any medium, provided the original author(s) and source are credited.

\section{References}

1. Atkinson, A.B.: On the measurement of inequality. J. Econ. Theory 2, 244-263 (1970)

2. Blanchflower, D.G., Oswald, A.: Well-being over time in Britain and the USA. J. Public Econ. 88, 1359-1386 (2004)

3. Clark, A.E., Senik, C.: Who compares to whom? The anatomy of income comparisons in Europe. Working Paper n2008-65, CEPREMAP, Paris School of Economics (2009)

4. Clark, A.E., Frijters, P., Shields, M.A.: Relative income, happiness and utility: an explanation for the Easterlin paradox and other puzzles. J. Econ. Lit. 46, 95-144 (2008)

5. Dolan, P., Peasgood, T., White, M.: Do we really know what makes us happy? A review of the economic literature on the factors associated with subjective well-being. J. Econ. Psychol. 29, 94-122 (2008)

6. Easterlin, R.A.: Life cycle happiness and its sources: intersections of psychology, economics, and demography. J. Econ. Psychol. 27, 463-482 (2006)

7. Ferrer-i-Carbonell, A.: Income and well-being: an empirical analysis of the comparison. Income effect. J. Public Econ. 89, 997-1019 (2005)

8. Ferrer-i-Carbonell, A., Frijters, P.: How important is methodology for the estimates of the determinants of happiness? Econ. J. 114, 641-659 (2004)

9. Ferrer-i-Carbonell, A., Van Praag, B.M.S.: Income satisfaction inequality and its causes. J. Econ. Inequality 1, 107-127 (2003)

10. Frey, B.S., Stutzer, A.: What can economists learn from happiness research? J. Econ. Lit. 40, 402-435 (2002)

11. Graham, C.: The economics of happiness: new lenses for old policy puzzles. In: Durlauf, S., Blume, L. (eds.) The New Palgrave Dictionary of Economics, 2nd edn. Palgrave MacMillan, Hampshire (2008) 
12. Graham, C., Felton, A.: Inequality and happiness: insights from Latin America. J. Econ. Inequality 4, 107-122 (2006)

13. Hagenaars, A.J.M., van Praag, B.M.S.: A synthesis of poverty line definitions. Rev. Income Wealth 31, 139-153 (1985)

14. Kahneman, D., Krueger, A.B., Schkade, D.A., Schwarz, N., Stone, A.A.: A survey method for characterizing daily life experience: the day reconstruction method. Science 3 306(no. 5702), 1776-1780 (2004)

15. Kapteyn, A., Van Praag, B.M.S., Van Herwaarden, F.G.: Individual welfare functions and social reference spaces. Econ. Lett., Elsevier 1(2), 173-177 (1978)

16. Layard, R.: Happiness: Lessons from a New Science. Penguin, London (2005)

17. Luttmer, E.F.P.: Neighbors as negatives: relative earnings and well-being. Q. J. Econ. 120, 9631002 (2005)

18. Plug, E.S., Van Praag, B.M.S.: Family equivalence scales within a narrow and broad welfare context. J. Income Distrib. 4(2), 171-186 (1995)

19. Senik, C.: When information dominates comparison. Learning from Russian subjective panel data. J. Public Econ. 88, 2099-2133 (2004)

20. Senik, C.: Direct evidence on income comparisons and their welfare effects. J. Econ. Behav. Organ. 72, 408-424 (2007)

21. Stutzer, A.: The role of income aspirations in individual happiness. J. Econ. Behav. Organ. 54, 89-109 (2004)

22. Van Praag, B.M.S.: The individual welfare function and its offspring. In: Cramer, J.S., et al. (eds.) Relevance and Precision, pp. 263-293. North-Holland, Amsterdam (1976)

23. Van Praag, B.M.S.: The perception of welfare inequality. Eur. Econ. Rev. 10, 189-207 (1977)

24. Van Praag, B.M.S.: Reflections on the theory of individual welfare functions. In: Proceedings of the American Statistical Association (1981)

25. Van Praag, B.M.S., Ferrer-i-Carbonell, A.: Happiness Quantified: A Satisfaction Calculus Approach (revised edition). Oxford University Press, Oxford (2004,2008)

26. Van Praag, B.M.S., Kapteyn, A., van Herwaarden, F.G.: The definition and measurement of social reference spaces. Neth. J. Sociol. 15, 13-25 (1979)

27. Wunder, C., Wiencierz, A., Schwarze, J., Küchenhoff, H., Kleyer, S., Bleninger, P.: Well-being over the life span: semiparametric evidence from British and German longitudinal data. IZA Dp No. 4155 (2009) 